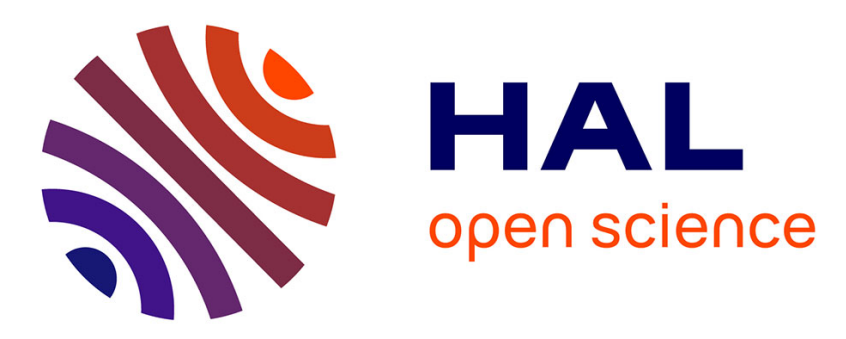

\title{
Critical point network for drainage between rough surfaces
}

Olivier Amyot, Frédérique Flukiger, Sandrine Geoffroy, Franck Plouraboué, Marc Prat

\section{- To cite this version:}

Olivier Amyot, Frédérique Flukiger, Sandrine Geoffroy, Franck Plouraboué, Marc Prat. Critical point network for drainage between rough surfaces. Transport in Porous Media, 2007, vol. 70, pp. 257-277. 10.1007/s11242-007-9098-3 . hal-00959368

\section{HAL Id: hal-00959368 https://hal.science/hal-00959368}

Submitted on 14 Mar 2014

HAL is a multi-disciplinary open access archive for the deposit and dissemination of scientific research documents, whether they are published or not. The documents may come from teaching and research institutions in France or abroad, or from public or private research centers.
L'archive ouverte pluridisciplinaire HAL, est destinée au dépôt et à la diffusion de documents scientifiques de niveau recherche, publiés ou non, émanant des établissements d'enseignement et de recherche français ou étrangers, des laboratoires publics ou privés. 


\title{
Critical point network for drainage between rough surfaces
}

\author{
O. Amyot · F. Flukiger - S. Geoffroy - F. Plouraboué • \\ M. Prat
}

\begin{abstract}
In this paper, we present a network method for computing two-phase flows between two rough surfaces with significant contact areas. Low-capillary number drainage is investigated here since one-phase flows have been previously investigated in other contributions. An invasion percolation algorithm is presented for modeling slow displacement of a wetting fluid by a non wetting one between two rough surfaces. Short-correlated Gaussian process is used to model random rough surfaces. The algorithm is based on a network description of the fracture aperture field. The network is constructed from the identification of critical points (saddles and maxima) of the aperture field. The invasion potential is determined from examining drainage process in a flat mini-channel. A direct comparison between numerical prediction and experimental visualizations on an identical geometry has been performed for one realization of an artificial fracture with a moderate fractional contact area of about 0.3. A good agreement is found between predictions and observations.
\end{abstract}

Keywords Invasion percolation · Fracture $\cdot$ Two-phase flow $\cdot$ Drainage $\cdot$ Micro-models Small capillary number $\cdot$ Lubrication $\cdot$ Rough surfaces

\section{Nomenclature}

$\begin{array}{ll}g & \text { Gravity acceleration } \\ h(x, y) & \text { Local aperture field between surfaces } \\ h_{0} & \text { Local aperture of critical points } \\ h_{\infty} & \begin{array}{l}\text { Local aperture associated with the interface whose posi- } \\ \text { tion along coordinate } y \text { is } \mathrm{y}_{\infty}\end{array} \\ \mathrm{H}=\partial_{i j}^{2} h & \text { Is the local Hessian matrix of the aperture field }\end{array}$




\begin{tabular}{|c|c|}
\hline$H_{x x} \& H_{y y}$ & Are the Hessian principal eigenvalues \\
\hline$\ell$ & Local width of the saddle-point throats \\
\hline$\ell_{\text {cap }}$ & Capillary length \\
\hline$\ell_{c}$ & Spacial correlation length of aperture field \\
\hline$L$ & Total width of the micro-channel \\
\hline$\Delta P$ & Difference of pressure between gas and liquid \\
\hline$Q$ & Volumetric flow rate per unit width \\
\hline$R_{i}, i=1,2$ & Are the principal radii of curvature of the interface \\
\hline$R_{0}$ & $\begin{array}{l}\text { Is the in-plane radius of curvature of the interface at the } \\
\text { origin } O\end{array}$ \\
\hline$y(x)$ & $\begin{array}{l}\text { Is the in-plane (i.e., }(\mathrm{x}, \mathrm{y}) \text { plane) position of the interface } \\
\text { in direction } y \text { at coordinate } x\end{array}$ \\
\hline $\mathrm{y}_{\infty}$ & $\begin{array}{l}\text { Is the in-plane (i.e., }(\mathrm{x}, \mathrm{y}) \text { plane) asymptotic position of the } \\
\text { interface in direction } y\end{array}$ \\
\hline$\beta, \beta^{\prime}$ & $\begin{array}{l}\text { Local angle associated with the slope of the upper and } \\
\text { lower surfaces }\end{array}$ \\
\hline$\epsilon$ & $\begin{array}{l}\text { Small parameter associated with the aspect ratio of the } \\
\text { critical points: local aperture divided by width }\end{array}$ \\
\hline$\epsilon^{\prime}$ & Small parameter associated with the surface slopes \\
\hline$\kappa$ & Mean curvature of the interface \\
\hline$\mu$ & Dynamic viscosity of the fluid \\
\hline$\rho$ & Fluid density \\
\hline$\sigma_{i}$ & Is the root mean square roughness of surface $i=1,2$ \\
\hline$\sigma=\sqrt{\sigma_{1}^{2}+\sigma_{2}^{2}}$ & Is the composite roughness \\
\hline$\theta, \theta^{\prime}$ & Wettability angles \\
\hline
\end{tabular}

\section{Introduction}

Confined flows between rough surfaces are of interest to many geologic and engineering applications Adler and Thovert (1999), Sahimi (1995), National Research council (1996), Evans et al. (2001), Faybishenko et al. (2000), Berkowitz (2002). Not only they are of special importance in petroleum engineering or sub-surface hydrology, as frequently mentioned, but also they play a crucial role in tightness problem, an application that has motivated the present study. More specifically, we refer to metallic static gaskets, which are used extensively as primary seals in rocket engines or in the primary circuit of nuclear plants where severe conditions such as extreme temperatures impose full metallic parts instead of classical elastomer O-rings. Despite their technological importance, studies on static metallic gaskets are scarce in the published literature, e.g., Polycarpou and Etsion (2000), Geoffroy and Prat (2004), and references therein. In these systems, tightness is obtained by pressing two metallic surfaces one onto the other. However, the surfaces are rough (owing to machining). Therefore, roughness offers possible passages for the fluid if the applied load is not sufficient to reach the geometrical percolation threshold. Leakage tests of these systems are generally made using Helium gas as a fluid. This is why most of the flow studies in this context focus on single-phase flow. However, the leak in the applications involves other fluids and generally occurs through a liquid-vapor phase change process owing to the thermodynamic conditions on both sides of gasket. In this context, the study of two-phase flows initiated in the present article can be seen as a necessary first step toward the more involved case of vaporization. 
As in several previous studies, Mourzenko et al. (1996), Plouraboué et al. (2001) we consider short-correlated rough surfaces. This class of random rough surfaces has for example been proposed to model rough cracks, while using Gaussian-correlated aperture fields (see Adler and Thovert 1999, and references therein). It might nevertheless be argued that many fractures are generally self-affine over a large range of scales rather than simply short-range correlated. However, even if cracks have been found to be long-range correlated surfaces Brown and Scholz (1985), a typical correlation length can be found in fractures because of the relative displacement between the two faces of the crack Plouraboué et al. (1995), Loggia et al. (1995) or other physico-chemical mechanisms Adler and Thovert (1999), Glass et al. (2001).

Hence, Gaussian fractures can be constructed so as to possess some important characteristics of most real fractures, such as small local slopes and short-range correlated aperture field. It can also be noted that studies of two-phase flows in fractures are not so numerous and suffer from various limitations, e.g., Glass et al. (2001). The modeling is often performed by mere adaptation of models previously elaborated in the context of porous media Vandersteen et al. (2003), Fourar et al. (1993). Thus the specificity of fracture's geometry is generally omitted in those analyzes. Hence, one of the objective of this paper is precisely to take into account the peculiar characteristics of fracture's for short-range correlated rough surfaces. This study is based on a representation of the aperture field as a random network of bonds. Though pore network models have become a classic modeling approach in the related field of transport in porous media, e.g., Sahimi (1995), Pereira et al. (1996), Blunt (2001), Blunt et al. (2002), Sok et al. (2002) it seems that our pore network model is the first one especially designed for the study of flows between rough surfaces in contact. As pointed out by Bryant et al. (1993), Blunt et al. (2002) most of pore network models are based on a regular lattice, typically a square or a cubic lattice, whereas a porous medium has generally an irregular structure. Though this can be acceptable in order to get insight into complex transport phenomena, such as two-phase flows for example, it is desirable to develop more adequate description of the pore space in order to study real porous media Sok et al. (2002). However, finding an equivalent skeleton to describe the network is non-trivial. In addition, even when a network can be described so as to preserve some topological properties of the real structure, it is not always obvious to assign accurate local transport properties to the pores and throats forming the network. In this context, the pore network model presented in this paper has several distinguishing features compared to standard lattices :

i. Its topology is based on the direct identification of geometrical features of random rough fractures: the geodesics of the aperture field's critical points. These objects play a key role in the analysis of transport phenomena under consideration.

ii. The local conductivities associated with the bond of this random discrete network are asymptotically exact. More precisely, the network model that is built can be formally regarded as the asymptotic limit of the transport problems on the continuum formed by the pore space, which is the aperture field in our case.

iii. Since our method permits a direct mapping of a complex random fracture onto a discrete network, it can be used for a direct comparison between experimental measurements in random fractures and theoretical prediction. This is one important contribution of our analysis that we were not able to find elsewhere.

This geodesic based pore network has been presented by Plouraboué et al. (2006) for the investigation of single phase flow in rough fractures. It has also permitted the study of the hydraulic and electrical percolation conductivity exponents by Flukiger et al. (2006). The relevance of such a network for modeling two-phase flows in random rough fracture in con- 
tact is the purpose of the present study. Referring to the large number of studies devoted to two-phase flows in porous media, it can be anticipated that the modeling of a given two-phase flow will be more or less difficult depending on the nature of the displacement, i.e., imbibition or drainage, and the possible unsteadiness of the flow, e.g., Fourar et al. (1993). In the present study, we address one of the simplest displacement process, drainage, at very low capillary numbers, i.e., the very slow immiscible displacement of a wetting fluid by a non-wetting one. It is now well established that drainage in porous media in the quasi-static limit is well described by invasion percolation (IP), Wilkinson and Willemsen (1983), De Gennes (1983), Wilkinson (1984), Lenormand et al. (1988). Furthermore, recent works indicate that IP concept is also useful when modeling and analyzing liquid-vapor phase change in porous media, such as slow drying, (see for example, Prat 2002) or the slow growth of bubbles driven by heat or mass transfer, e.g., Satik and Yortsos (1996), Satik et al. (1995), Bories and Prat (2002). Thus it may be anticipated that a better understanding of slow drainage in fracture could be useful for the study of these processes in rough fractures. Slow drainage in fractures has been the subject of several studies (see Glass et al. 2001, and references therein). Amundsen et al. (1999) studied slow drainage in two artificial fractures, one with a discrete self-affine aperture field (model A) and the other with a continuous aperture field, which was self-affine only below the typical size of main "valleys" (model B). It seems that these fractures were well connected, i.e., the contact fractional area between top and bottom plates was very small or null. Comparisons were made between experimental data and results of simulation based on variants of standard IP model for both fractures. The displacement in model A was simulated using the standard IP model (see below) with simultaneous invasion of all sites with lowest threshold. Because of snap-off events and redistribution of non-wetting fluid observed in model B, a more complex variant of IP model was tested for model B. As these events are not frequent in our case, this model does not need to be detailed here, but the interested reader could consult Amyot and Plouraboué (2007) for a thorough investigation of capillary snap-off in similar constrictions. Amundsen et al. found that their model led to a reasonable agreement with the experimental data. Interestingly, it was found that the more complex IP model for model B led to poorest results than the IP model with simultaneous invasion when applied to model A. Thus this study suggests that the IP model should be modified depending on the characteristics of each fracture. The standard IP model for fracture mentioned earlier can be described as follows. The fracture aperture field is simply conceptualized as a planar checker board of individual elements with a four-fold connectivity, the center of each element with a known local aperture. The invasion pressure of an element is simply defined as

$$
P_{t h}=\frac{2 \gamma \cos \theta}{h}
$$

in which $h$ is the element local aperture, $\gamma$ is the surface tension, $\theta$ the contact angle. The algorithm essentially consists of repeating the following operation: invade the adjacent element to the invaded region that has the lowest invasion pressure by filling it with invader fluid. Examples of studies in which this model is used are those of Mendoza and Sudicki (1991), Wagner et al. (1996), Vandersteen et al. (2003). It also corresponds to model A of Amundsen et al. (1999). Glass et al. (1998) pointed out that the standard IP model does not take into account the influence of local in-plane curvature of the fluid/fluid interface. They develop a modified IP model taking into account the in-plane curvature with an ad hoc model and made comparisons with experiments. They found that the local in-plane curvature can greatly affect the phase invasion structure. In contrast with seemingly all previous studies related to drainage in fracture, we focus on fractures with significant contact areas between top and bottom. In the latter we call this type of fracture, "sparsely connected" (which is the 
case of interest for the tightness problem evoked above). Moreover, rather than developing a site IP model directly based on the local aperture field (as done in all previous studies), we develop a bond IP model that is built on a bond/site network. Thus, we retrieve a feature of porous media network models, i.e., a representation of the pore (here the aperture) space in terms of bond (throat)/site (pore) network. This network model is determined from the fracture surfaces geometry. It has common features with the equivalent network introduced by Weinrib (1982) for studying the percolation threshold of a class of two-dimensional continuum systems. As in many pore network models of quasi-static drainage (e.g., Blunt et al. 2002 , for a review and numerous references) the non-wetting fluid must overcome the capillary pressure threshold associated with a bond for the invasion to proceed. Thus, in addition to obtain an appropriate representation for the aperture field in terms of network, this approach relies on a correct estimation of bond capillary pressure thresholds. In this respect, the present paper relies on a previous work, by Geoffroy et al. (2006), devoted to the study of drainage in elementary flat straight channels. In the latter, an asymptotic analytical expressions for the pressure difference across a meniscus traveling in the channel in the quasi-static limit is derived. The saddle-points considered in the present study have locally a geometry similar to one of the channel shapes considered in Geoffroy et al. (2006). Hence, the invasion criteria previously obtained for straight channels is extended through an asymptotic analysis to saddle-points of interest for the present study. In particular, we show how the capillary pressure threshold depends on the fluid/fluid in-plane curvature. Furthermore, this study presents the first direct comparison between local IP rules obtained on saddle-points, and experimental observations in a similar numerically generated random fracture. Finally, as indicated previously, the present paper can be considered as a follow-up of the paper by Plouraboué et al. (2006), which was devoted to the computation of single-phase flow in similar systems as those considered here for the study of drainage.

As we shall see, the capillary pressure threshold depends on the fluid/fluid in-plane curvature and is studied here through an asymptotic analysis.

The paper is organized as follows. We start with the network construction in Sect. 2. In Sect. 3, we discuss the invasion of a single bond and discuss the resulting capillary pressure threshold. In Sect. 4.1, we describe the drainage algorithm. Comparisons between simulations and experiments will be presented in Sect. 4.3.

\section{Network representation of short-correlated aperture field}

\subsection{Gaussian fracture}

The system under study is sketched in Fig. 1. For simplicity in presentation, the top surface (surface 2) is assumed to be flat and smooth whereas the bottom surface (surface 1) is rough. This choice is not restrictive, and all the presented analysis can straightforwardly be transposed in the case of two rough surfaces, as far as the slope of the rough surface remains small enough. More precisely, it is possible to establish the equivalence of flow transport between two rough surfaces of respective root mean square (r.m.s) roughness $\sigma_{1}$ and $\sigma_{2}$ to the flow within an equivalent single rough surface of effective roughness $\sigma=\sqrt{\left(\sigma_{1}^{2}+\sigma_{2}^{2}\right) / 2}$ and a flat plane. This is called a "composite approximation" in the context of lubricated flows Letalleur et al. (2002). It can be shown that this approximation is consistent with lubrication approximation associated with the flow since the neglected geometrical effects coming from the surface tortuosity are of order $O\left(\epsilon^{\prime 2}\right)$ Prat et al. (2002) where $\epsilon^{\prime}$ is the typical amplitude 


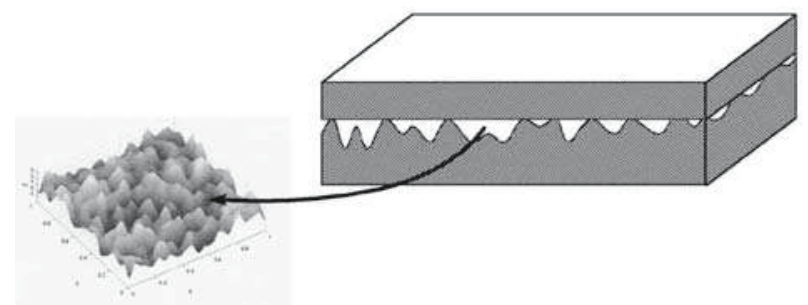

Fig. 1 Schematic view of the rough surfaces under study

of the local slope of both rough surfaces which is generally a small parameter. One may wonder at this stage whether it is still valid to consider the equivalent system formed by the flat surface and the rough composite surface for two-phase flow drainage between two rough surfaces. As shown in the Appendix, the general problem of drainage between partially wetting rough surfaces can be analyzed in this context. The invading criteria associated with the local curvature of the interface can be exactly mapped onto those obtained between a rough and a plane surface. Therefore the equivalent system can be considered for drainage as well.

In the next section we discuss the relation between the discrete network built from a continuous description of surface roughness and lubrication approximation. The upper and lower rough surfaces are then described through their respective surface height $z_{i}(x, y)$, with $i=1$ for the bottom surface and $i=2$ for the top one. Since, the upper surface is flat, its height $z_{2}$ does not depend on $x$ and $y$. We furthermore consider in this paper a special class of random rough surfaces: those having short-range correlation, that is those belonging to the Markovian family of stochastic surfaces. More precisely, we have considered a surface roughness model which fulfills an isotropic Gaussian covariance function:

$$
\left\langle\left(z_{1}\left(\mathbf{r}^{\prime}\right)-z_{1}\left(\mathbf{r}^{\prime}+\mathbf{r}\right)\right)^{2}\right\rangle_{\mathbf{r}^{\prime}}=\sigma^{2}\left(1-e^{-\left(\frac{r}{l_{c}}\right)^{2}}\right),
$$

where $\mathbf{r}^{\prime}, \mathbf{r}$ are vectors in the $(x, y)$ plane, $r$ is the norm $r=\|\mathbf{r}\|$, and $l_{c}$ is the correlation length. It shall be noted that the proposed analysis has a general scope and does not depend on this precise choice for the short-range correlation model. Although other choices have been used in the literature, e.g., Chrysikopoulos and Abdel-Salam (1997), Chrysikopoulos and James (2003), the Gaussian covariance model has been chosen for a particular example was needed. It is worth noting however that its relevance for fractures has been previously considered Adler and Thovert (1999). In addition we note that the choice of a Gaussian covariance function leads to a $\mathcal{C}^{2}$ continuous aperture fields, which is needed for computing the second derivatives of aperture field (see Sect. 2.2). The local distance $d(x, y)$ between the two surfaces is then $d(x, y)=z_{2}-z_{1}(x, y)$. As in several previous works, e.g., Adler and Thovert (1999), Prat et al. (2002), the local aperture field $h(x, y)$ is then simply defined by

$$
\begin{array}{cl}
h(x, y)=d(x, y) & \text { if } \mathrm{d} \geq 0 \\
h=0 & \text { if } \mathrm{d}<0 .
\end{array}
$$

The zones where the local distance is negative, i.e., $d<0$, are zones of local contact between the two surfaces. The presented analysis could be extended to more sophisticated fracture's models for which the aperture field can be influenced by elastic deformation. We 
nevertheless restrict our point of view to this simple model to better control the experimental geometry in order to test a bond IP model.

\subsection{Bond network of critical points}

Figure 1 shows an example of Gaussian correlated rough surface (surface 1). One can distinguish the valleys, which are the regions of minimum height, the peaks, which are the regions of maximum height and the saddle-points, which are the points of minimum height between two valleys. Similarly, in the absence of contact between the two solid surfaces, one can define the critical points of the aperture field as the points where $\nabla h=\mathbf{0}$. These points correspond to the minima, saddle-points, and maxima of the aperture field. When the two surfaces are in contact, contact areas appears at the minima of the aperture field (which correspond to surface 1 maxima). Thus, as far as the aperture space is concerned, we are primarily interested in the saddle-points and maxima. The saddle-points are the points where $\nabla h=\mathbf{0}$ and det $\mathbf{H}<0$, where $\mathbf{H}$ is the Hessian matrix whose coefficients are related to the second derivative of the aperture $h$ :

$$
H_{i j}=\partial_{i j}^{2} h,
$$

where $i, j=1$ refers to coordinate $x$ and $i, j=2$ refers to coordinate $y$. The maxima are the points where $\nabla h=\mathbf{0}$, det $\mathbf{H}>0$ and $\operatorname{Tr} \mathbf{H}=\left(\partial_{x}^{2} h+\partial_{y}^{2} h\right)<0$. Those simple criteria permit to identify the maxima and saddle-points. From these one can construct the network by determining the paths from maximum to maximum through the saddle-points as detailed by Plouraboué et al. (2006). This network is interesting to consider for fluid transport because, most of the viscous pressure drop between two adjacent maxima is located in saddle-points. More precisely, using an asymptotic analysis similar to the one used for the usual lubrication approach, one can find that the pressure drop is controlled by the local aperture and the Hessian matrix eigenvalues at the saddle-point Plouraboué et al. (2004). Moreover, when considering two-phase flow, each saddle-point is a constriction where the aperture is minimal along the continuous path linking two maxima. Hence, the capillary pressure is maximal at the saddle-point. Then the capillary pressure not only depends on the local aperture of the saddle-point, but also on its geometry, i.e., on the local value of the Hessian matrix. This point will be further considered in the next section.

The network is connected to the boundary of the fracture through the steepest ascent paths initiated at the saddle-points located near the boundary. The random rough surface is generated in Fourier space while prescribing a short-correlated spectral representation (see for example Makse et al. 1996). This method gives a discrete field of heights on a Cartesian $N \times N$ regular grid. The gradient of $h$ as well as the Hessian matrix can be computed in Fourier space at each grid nodes, so that the critical points can be found (cf. Plouraboué et al. 2006, for more details). The bonds between aperture maxima are then constructed with a steepest ascent method. An example of aperture field with its network is shown in Fig. 4. When there is a significant contact area between the two surfaces, the network contains a distribution of bond clusters. The fluid displacement takes place on the percolation cluster only. To identify the bonds belonging to the percolation cluster, the depth first search method Tarjan (1983) has been implemented. The last step consists in identifying the skeleton of the percolation cluster. 

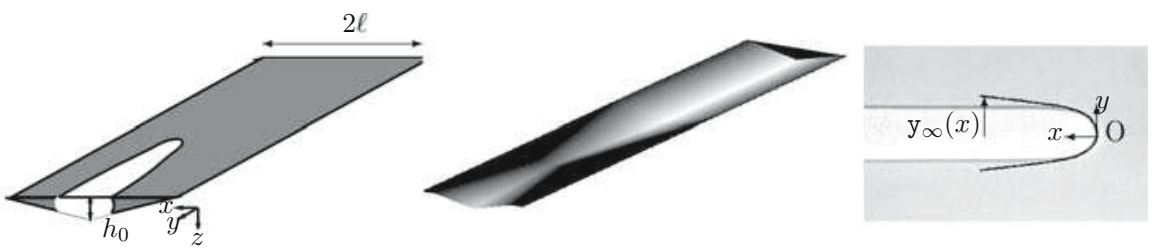

Fig. 2 (a) Sketch of flat straight linear channel with $h_{0}=1 \mathrm{~mm}, \ell=25 \mathrm{~mm}$. (b) Perspective view of a linear constricted channel. (c) Top view showing the comparison between the experimental bubble invading channel depicted in Fig. 2a with gray line and Fig. $2 \mathrm{~b}$ in black line

\section{Bond capillary pressure threshold through a single saddle-point}

\subsection{Theoretical discussion}

As in any bond invasion percolation model on a network, an invasion threshold has to be assigned to each bond. According to Laplace's law, at zero capillary number limit, the capillary pressure threshold associated with a perfectly wetting liquid reads

$$
P_{t h}=\gamma\left(\frac{1}{R_{1}}+\frac{1}{R_{2}}\right)
$$

where $R_{1}$ and $R_{2}$ are the main radii of curvature of the non-wetting/wetting fluids interface and $\gamma$ is the interfacial surface tension. The generalization of the forthcoming analysis to more general static wetting conditions is discussed in the appendix. The capillary pressure threshold is associated with the narrowest passage between two maxima. In our geometry, this corresponds to the constriction associated with the saddle-point located along the bond joining two aperture maxima. Thus we have to determine the capillary pressure needed to invade such constriction. Because of the gentle local slopes characterizing the rough surface, it can be surmised that the principal curvature directions of the liquid-gas interface are oriented either in a vertical plane (parallel to the $z$ direction) and in the $(x, y)$ plane. In the following we will often call $(x, y)$ variations the "in-plane" variations of the interface. Moreover, since, the vertical dimension is always smaller than the horizontal ones, the local interface shape along any vertical plane is a semi-circle whose radius fits the local distance between the two solid surfaces, assuming again a perfectly wetting liquid. Hence the radius of curvature along the $z$ direction of the interface at a given $(x, y)$ position is $h(x, y) / 2$. This radius of curvature is always smaller than the in-plane radius of curvature, so that the pressure jump is mainly controlled by this term. Hence the first radius of curvature $R_{1}$ of relation (5) is the local half-distance between the two surfaces, i.e., $R_{1}=h(x, y) / 2$.

Nevertheless one needs to go beyond the first-order estimate (1) for the capillary pressure jump. We are interested in finding invasion criteria which could distinguish between the invasion of two saddle-points having the same local aperture, but different shapes. Again, because of small surface slopes, the liquid/gas interface within the saddle-point constriction remains close to the saddle-point central position as illustrated in Figs. 2a and c. The shape of the interface that is found for those constricted channels is very similar to those obtained in a straight channel by Geoffroy et al. (2006) (such as the one depicted in Fig. 2a) having the same cross-section as the saddle-point cross-section.

This qualitative view is more precisely argued in the following. Quasi-static drainage in narrow straight channels has been investigated by Geoffroy et al. (2006) in both linearly and quadratically varying channel cross-sections. These narrow channels have small aspect ratio. 
This means that the ratio between the maximum gap $h_{0}$ at the saddle-point and the channel's width $\ell$, is a small parameter $\epsilon=h_{0} / \ell \ll 1$. We would like to discuss here how the results that have been obtained by Geoffroy et al. (2006) can be transposed to find the saddle-point percolation geometrical criteria. Let us first consider the vicinity of a saddle-point. Since, saddle-points are critical points, they have quadratic shape in their vicinity, so that a Taylor expansion of the aperture field near any critical point reads:

$$
h(x, y)=h_{0}+\frac{1}{2} H_{y y} y^{2}+\frac{1}{2} H_{x x} x^{2}+\cdots
$$

where $h_{0}$ is the aperture at saddle-point $O$ taken as origin $\left(x_{O}, y_{O}\right)=(0,0)$, and $x$ and $y$ are the principal directions of the Hessian matrix at the saddle-point which are orthogonal. The Hessian eigenvalues $H_{x x}>0$ and $H_{y y}<0$ have opposite sign at the saddle-point. In the following we consider geometrical constrictions having the same order of magnitude for the Hessian eigenvalues i.e.,

$$
\beta=-H_{x x} / H_{y y} \sim O(1) .
$$

This expansion is valid for $x \& y$ positions smaller than $1 / H_{x x} \& 1 / H_{y y}$, respectively. We now consider the problem of quasi-static position of a non wetting/wetting fluid interface in such saddle-point. In the following we will denote y the position of the interface along the $y$ coordinate.

We first need to identify the important length-scales of the problem. To that purpose let us discuss the physical ingredient of the problem. In the limit of extremely slow displacement (zero capillary number limit) the pressure difference between the non-wetting and the wetting fluid is spatially uniform. Then, the interface mean curvature is spatially uniform. The static interface shape problem is thus a free-boundary value problem associated with a constant mean curvature $\kappa$. The unknown value $\kappa$ is fixed by the channel shape. Hence, depending on the channel shape at the origin $O$, different scalings can be obtained for the typical width of the invading air bubble Geoffroy et al. (2006). Let us discuss here the case of quadratic channel for which the aperture has a quadratic shape (6). Far from the origin (in a sense that will be precised below) the interface position $y_{\infty}$ follows a constant level-set $h_{\infty}$ of the channel aperture, where the mean curvature is obviously constant. Hence, from (6) the associated far field interface position $\mathrm{y}_{\infty}\left(h_{\infty}, x\right)$ depends on the longitudinal coordinate $x$, through relation:

$$
h_{\infty}=h_{0}+\frac{1}{2} H_{y y} y_{\infty}^{2}+\frac{1}{2} H_{x x} x^{2}
$$

In the case of a straight channel, with no longitudinal dependence (i.e., $H_{x x}=0$ in (8)), the interface position far from the origin $\mathrm{y}_{\infty}$ is obviously spatially constant. Let us now discuss the relationship between the far-field constant level-set $h_{\infty}$ and the mean interface curvature. First, one has to consider the transverse dimensions $\ell$, associated with the channel width at the saddle-point. Given (6), this width is $\ell=\sqrt{-2 h_{0} / H_{y y}}$, because at a transverse distance $\ell$ the aperture gap is zero at the saddle where $x=0$. One has then to realize that the geometrical radius of curvature $-1 / H_{y y}$ is then much larger than $\ell$, i.e.,

$$
-1 / H_{y y} \sim \ell / \epsilon
$$

Moreover, since, from the definition (7) of $\beta$, it is an order one parameter, the same is true for the longitudinal curvature,

$$
1 / H_{x x} \sim \ell / \epsilon .
$$


In the "far-field" region where the interface reaches a constant level-set $h_{\infty}$ the interface radius of curvature in the $(x, y)$ plane are directly related to the surface radius of curvatures $1 / \mathrm{H}_{x x} \& 1 / \mathrm{H}_{y y}$ (in the case of surfaces having small slopes, those quantities are indeed the principal radius of curvature of the solid surfaces). This is not the case near the saddle-point, where the in-plane interface radius of curvature at the origin $R_{0}$ is directly related to the channel width $\ell$. Since, the in-plane variations of the interface shape are much larger than $\ell$ when following the aperture isovalues, the in-plane interface curvature far from the origin is then negligible compared to the in-plane curvature at the origin $1 / R_{0}$. Hence, the total mean curvature $\kappa$ of the far-field interface position of the liquid/air interface can be approximated by its $z$ contribution:

$$
\kappa \sim 2 / h_{\infty}
$$

which is exactly identical to the curvature at the finger tip origin $O$, since the mean curvature is spatially constant in the quasi-static regime. The curvature at point $O$ is the sum $2 / h_{0}+1 / R_{0}$ where, again, $R_{0}$ is the in-plane (along the $x y$ plane) radius of curvature at the origin. Hence,

$$
1 / R_{0} \sim 2 / h_{0}-2 / h_{\infty} .
$$

One thus needs to find how close $h_{\infty}$ is to $h_{0}$ to get an estimate of how much the in-plane curvature contribute to the mean curvature. To find this, one has to realize that even if $\mathrm{y}_{\infty}(x)$ is longitudinally varying, it is nevertheless always smaller than the local channel width, as can be observed experimentally. For the same reason that a narrow gap produce a narrow bubble, the asymptotic far field isovalue $h_{\infty}$ is also closed to $h_{0}$, so that

$$
\left(h_{\infty}-h_{0}\right) / h_{0} \sim \epsilon^{2 \alpha},
$$

where $0<\alpha<1$ is yet to be found. Using this relation, we deduce from (8), that

$$
\left(y_{\infty}^{2}-\beta x^{2}\right) \sim \epsilon^{2 \alpha} h_{0} /\left|H_{y y}\right| \sim \epsilon^{2 \alpha} \ell^{2} .
$$

This relation then leads to a new length-scale $w=\epsilon^{\alpha} \ell$ which is in between $h_{0}$ and $\ell$, so that

$$
\left(y_{\infty}^{2}-\beta x^{2}\right) \sim w^{2} .
$$

The same relation $\left(h_{\infty}-h_{0}\right) / h_{0} \sim \epsilon^{2 \alpha}$ gives the in-plane curvature at the origin from

$$
1 / R_{0} \sim 2 / h_{0}-2 / h_{\infty} \sim 2\left(h_{\infty}-h_{0}\right) / h_{0} h_{\infty} \sim \epsilon^{2 \alpha} 2 / h_{0},
$$

so that,

$$
R_{0} \sim \epsilon^{-2 \alpha} h_{0} / 2 \sim \ell \epsilon^{1-2 \alpha} .
$$

This relation shows that the in-plane interface radius of curvature at the origin $O$ is smaller than $\ell$ as far as $\alpha<1 / 2$. At a distance $w$ from the origin, the interface gently reaches its asymptotic shape $y_{\infty}$ following the channel aperture isovalue $h_{\infty}$, so that the in-plane curvature is well approximated by the second derivative of the transverse shape

$$
1 / R \sim y_{\infty} / x^{2} \sim 1 / w \sim 1 / \epsilon^{\alpha} \ell \sim \epsilon^{1-\alpha} / h_{0} .
$$

Since, once more, the aperture isovalue $h_{\infty}$ is very close to the one at the origin $h_{0}$, any intermediate value of the aperture height $h(w, y)$ reached by the interface at distance $w$ from the origin is also closed to $h_{0}$. Then the scalings of the in-plane radius of curvature $R$ and $R_{0}$ with the small parameter $\epsilon$ should be identical. Using the previous estimates,

$$
R \sim \epsilon^{\alpha} \ell \sim R_{0} \sim \epsilon^{1-2 \alpha} \ell
$$


leads to $\alpha=1-2 \alpha \Rightarrow \alpha=1 / 3$ for a quadratic shape narrow channel. Hence, the exponent $\alpha$ previously introduced, gives the relation between the asymptotic aperture isovalue $h(x, y)=h_{\infty}$ and the aperture at the saddle-point $h_{0},\left(h_{\infty}-h_{0}\right) \sim h_{0} \epsilon^{2 / 3}$. This asymptotic isovalue $h_{\infty}$ is reached when the distance along $x$ is large compared to $w=\epsilon^{1 / 3} \ell$. This is precisely the "far-field" condition that we were using in the beginning of this analysis for which we have now found a precise formulation i.e., $w \ll x<1 / H_{x x}$. From this, one can also find the scaling of the in-plane radius of curvature at the origin i.e., $R_{0} \sim \ell \epsilon^{1 / 3}$. This confirms that $R_{0}$ is much smaller than $\ell$, which is in turn much smaller than $1 / H_{x x}$ $\& 1 / H_{y y}$. This is self-consistent with our previous statement that the in-plane curvature at the origin is much larger than in the far-field. This in-plane curvature of the interface tip is further investigated experimentally in the next section which confirms the fact that in-plane interface curvature in the far-field has a negligible contribution to the mean curvature. The obtained scalings are exactly similar with those previously found by Geoffroy et al. (2006) in the case of straight channels having quadratic shape. A more involved analyzes needs to be performed to find the prefactors of these scaling laws. Skipping the details of this analysis which closely follow the one presented by Geoffroy et al. (2006), it is possible to find that the asymptotic aperture isovalue $h_{\infty}$ reads:

$$
h_{\infty}=h_{0}\left(1-\left(\epsilon \frac{3 \pi}{16}\right)^{2 / 3}\right)+O\left(\epsilon^{4 / 3}\right)
$$

So that the pressure drop associated with the static capillary pressure in a saddle-point is easy to deduce:

$$
\begin{aligned}
& \Delta P=\frac{2 \gamma}{h_{\infty}}=\frac{2 \gamma}{h_{0}}\left(1+\left(\epsilon \frac{3 \pi}{16}\right)^{2 / 3}\right)+O\left(\epsilon^{4 / 3}\right) \\
& \Delta P=\frac{2 \gamma}{h_{0}}\left(1+\left(\frac{-h_{0} H_{y y} 9 \pi^{2}}{512}\right)^{1 / 3}\right)+O\left(\epsilon^{4 / 3}\right)
\end{aligned}
$$

From (10), one can see that the first contribution is related to the saddle-point minimum aperture $h_{0}$, as expected. The second term on the right hand side is related to the contribution of the in-plane radius of curvature, which only involves the transverse radius of curvature $-1 / H_{y y}$ of the saddle-point. It is important to note that this relation is not a model, but the result of an asymptotic expansion. This result has been compared to numerical and experimental results in straight channels by Geoffroy et al. (2006). Moreover it should be noted that the scaling argument that we have just developed for quadratic channels saddle-points could be equally applied for linear channels. More precisely the transposition of the results obtained by Geoffroy et al. (2006) for linear channel could equivalently be applied to locally linear constricted channels in both longitudinal and transverse direction and leads to a different exponent $\alpha$. Let us now discuss some experimental validation of this local analysis in the case of a constricted channel.

\subsection{Experimental analysis}

As illustrated in Fig. 2 we considered a narrow channel of maximum gap $h_{0}$ and total width $2 l$. The main point of this section is to show that the scaling argument developed in the previous section is confirmed by experimental observation. This argument claims that straight channel and constricted channel having equally small in-plane variations along the $x$ and $y$ directions leads to equivalent problems for quasi-static drainage. In other words, the 
longitudinal variations of the channel aperture $h(x, y)$ do not influence the interface quasistatic mean curvature so that the capillary pressure needed to invade the channel is controlled by the saddle-point width only, that is to say the transverse curvature only. To test this assumption we have compared the interface obtained in straight channels by Geoffroy et al. (2006) with a constricted channel with similar geometrical properties. More precisely, we have designed a channel for which the aspect ratio at the saddle-point is exactly the same as in the straight channel. Moreover, the $x$ and $y$ slopes of the aperture $h(x, y)$ have been chosen small and equal to $\epsilon=0.05$. In order to better observe the asymptotic limit where the interface reach the $h_{\infty}$ isovalue, we have chosen linear transverse and longitudinal variations of the aperture $h(x, y)$. In this case, when the interface aperture reach $h_{\infty}$, the interface shape tends to a straight-line. This linear behavior of the interface shape is thus much easier to detect experimentally than the parabolic interface shape which would have arisen in the quadratic saddle-point analyzed in the previous section. This is why we have preferred here to test the analysis presented in the previous section in a linear channel whose variation around the "saddle-point" $O$ is $h(x, y)=h_{0}(1+\epsilon(x-y))$.

To this end a mini-channel has been built in two steps. First, a polymeric material has been carved with a digitally controlled ISEL CPM4040 milling machine. The mini-channel depth function $h(x, y)$ has been very finely discretized, so as to permit an optimal use of the machining precision, which is equal to 10 microns in every $(x ; y ; z)$ directions. The mini-channel maximum depth $h_{0}$ has been chosen equal to $h_{0}=1 \mathrm{~mm}$. The maximum depth $h_{0}$ is only slightly smaller than the capillary length $l_{\text {cap }}=\sqrt{\gamma / \rho}$ obtained from balancing surface tension $\gamma$ with gravity acceleration $g$ acting on the fluid volume density $\rho$ $\left(l_{\text {cap }} \approx 1.5 \mathrm{~mm}\right.$ for the oil used in our experiments). Nevertheless gravity forces influence should remain small in the presented experimental results, since every experiment has been carefully aligned along the horizontal plane. In a second step, a Plexiglas plate of $1 \mathrm{~cm}$ thick is fixed on top of the carved material. Leakage is prevented thanks to a silicon paste disposed on lateral grooves machined along the carved channel. The top plate has been fixed with equally spaced and controlled clamping screws. Then, the entrance side of the channel is open to ambient pressure, while the exit side is connected to a screw-driven pump through a Plexiglas chamber. Experiments were carried out by slowly pumping out a perfectly wetting silicon oil $\left(\gamma=20.7 \times 10^{-3} \mathrm{~N} / \mathrm{m}\right)$ that perfectly wets the material of both top and bottom surfaces. A constant temperature of $22 \pm 1^{\circ} \mathrm{C}$ has been maintained during all the experiments.

The experiments were recorded with a CCD camera set above the channel. One can refer to Geoffroy et al. (2006) for further details on the experimental aspects. The channel geometry is illustrated in Fig. 2b. Two distinct parts can be distinguished. First, a straight channel with triangular cross-section and secondly a constricted linear section. The straight channel half-width is $\ell_{1}=20 \mathrm{~mm}$ and its length is $14 \mathrm{~cm}$. Thus its slope is $\epsilon=h_{0} / \ell_{1}=0.05$. The constriction is obtained by linear variation of the channel half width and maximum depth so as to keep the same slope in each cross-section. This variation occurs over a distance of $90 \mathrm{~mm}$ from the end of the straight channel. At the "saddle-point", the channel half-width is thus $\ell_{2}=5 \mathrm{~mm}$ and the maximum depth is $0.25 \mathrm{~mm}$. The geometry of the divergent after the saddle-point is symmetrical with the convergent. Figure $2 \mathrm{c}$ shows the superposition of liquid-air interface at the constricted saddle-point (black continuous curve) during drainage with the one traveling within the straight channel (gray curve). The latter has been rescaled by the ratio $\ell_{2} / \ell_{1}$ between the straight channel width and the saddle-point cross-section width. This rescaling is precisely the one obtained by Geoffroy et al. (2006), which shows that the dimensionless interface location (the ratio between the interface position and the local channel width) depends only on the aspect ratio $\epsilon$, which is kept constant all along the channel here. As can be observed, the superposition shown in Fig. 2c gives a perfect 
matching between the respective in-plane radius of curvature $R_{0}$ of the interface at the bubble tip $O$. Since, the mean curvature is constant in both cases, this indicates that the capillary pressure is also identical in a straight channel and in a longitudinally slowly varying channel. The interfaces obviously differ far from the bubble tip $O$ because they reach isovalue of the aperture field. The far field interface position $\mathrm{y}_{\infty}$ is constant for a straight channel. On the contrary it varies linearly in the linear constricted channel and reaches a straight line whose angle with the longitudinal direction is proportional to the aspect ratio $\epsilon$. In both cases, one can clearly distinguish that each asymptotic far field positions associated with isovalue $h_{\infty}$ is reached on a spatial scale which is much smaller than the channel width $\ell$. This observation is consistent with the finding of Geoffroy et al. (2006), as well as the analysis of the previous section for which the spatial scale associated with asymptotic convergence has been found equal to $w=\epsilon^{1 / 3} \ell$ which is much smaller than $\ell$.

\section{Invasion percolation between rough surfaces}

\subsection{Quasi-static drainage modeling}

Implementing IP algorithm in the present context is now straightforward. Initially, all bonds are occupied by the wetting fluid. The non-wetting fluid can invade the network from one of its four sides, say the bottom side. The algorithm then consists of repeating two steps:

1. Identify the bond adjacent to the invaded region that has the lowest invasion threshold

2. Invade the identified bond and fill all bonds connected to the adjacent maxima up to the saddle-points.

Two additional rules have been added to the previous ones,

3. bonds not belonging to the percolation cluster skeleton cannot be invaded

4. bonds belonging to trapped wetting fluid bond cluster cannot be invaded

The invasion stops when the non-wetting fluid forms a spanning cluster from bottom to top sides. Periodic invasion conditions are imposed on lateral sides of network. In the next section results of drainage simulation based on this algorithm are compared to experimental visualizations.

\subsection{Drainage experiments between micro-machined rough surfaces}

In order to check the IP network model, a numerically generated "random" rough surface has been constructed using the same digitally controlled milling machine. The statistical properties of the surface are isotropic and short-correlated, as previously discussed in Sect. 2. The surface discretization is $2 \pi$ points per correlation length $l_{c}$ while approximately 80 correlation lengths have been taken along each direction. The surface dimensions were set to $120 \times 120 \mathrm{~mm}$ with a vertical r.m.s roughness equal to $\sigma=1 \mathrm{~mm}$ while the correlation length is equal to $l_{c}=1.2 \mathrm{~mm}$. Then the aperture field is truncated so that every height larger than $h_{\text {max }}=0.55 \mathrm{~mm}$ are set to $0.55 \mathrm{~mm}$.

This generates plateaus corresponding to the contact areas. This surface is carved with the digitally controlled milling machine. A transparent flat Plexiglas plate one centimeter thick is fixed on top of the carved material using the same protocol described in previous section for the mini-channel. The channel entrance is open to ambient pressure, while the exit side is connected to a screw-driven pump through a Plexiglas chamber. Experiments are carried out by slowly pumping out a silicon oil that perfectly wets the material of both 
(a)

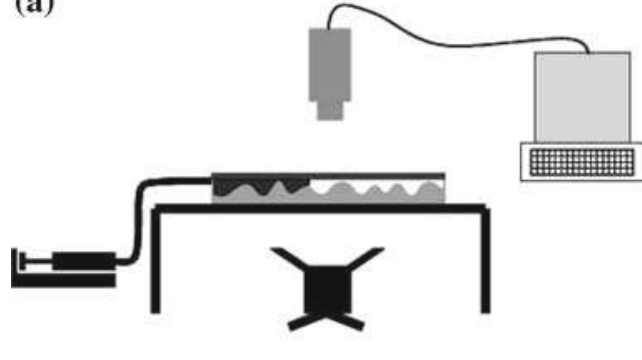

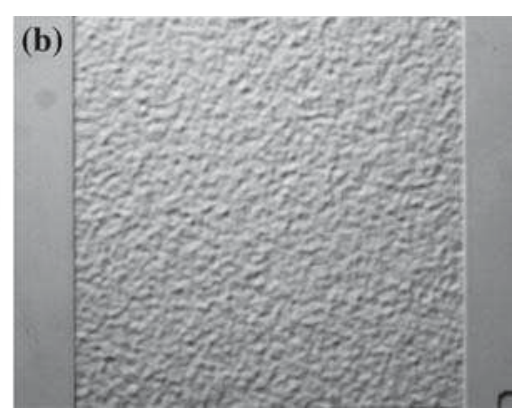

Fig. 3 (a) Schematic view of slow drainage experiment within the artificial fracture. (b) Top view of the milling machined artificial fracture

top and bottom surfaces. A constant temperature of $22 \pm 1{ }^{\circ} \mathrm{C}$ is maintained during all the experiments. The controlled flow rate imposed in the experiment is $Q=3.33 \times 10^{-10} \mathrm{~m}^{3} / \mathrm{s}$. Thus an average value of capillary number can be estimated as $C a=Q \mu / \bar{d} L \gamma$ where $\mu$ is the silicon oil dynamic viscosity $(\mu=0.068 \mathrm{Pas}), \bar{d}$ is the distance between the mean planes of both surfaces $(\bar{d}=450 \mu \mathrm{m})$ and $L$ is the artificial fracture width $(L=0.12 \mathrm{~m})$. This leads to $C a=2 \cdot 10^{-4}$ in agreement with the assumption of a quasi-static displacement. The experiments are recorded with a CCD camera set above the artificial fracture. Figure 3 shows a sketch of the experimental set-up as well as an image of the milling machined fracture. There are some unavoidable experimental uncertainties concerning the exact reproduction of the numerical aperture field coming from the machining precision, elastic deformations of the materials, the Plexiglas parallelism, etc. The experimental uncertainty coming from micro-machining is easy to estimate and is close to 30 microns. The other uncertainties coming from the possible elastic deformation of the top Plexiglas plate which is screwed over the polymeric material are more difficult to evaluate. A reasonable guess of the discrepancy between the experimental vertical aperture field and its numerical model should not be less than a hundred microns.

\subsection{Comparison between experiments and IP computation}

The drainage simulation has been performed using the IP algorithm described in Sect. 4.1. The network is constructed using the same numerically generated aperture field as for the experimental model described in the previous section for which the liquid-gas interface is geometrically controlled by the capillary pressure. As a matter of fact, the interface position shown in Fig. 4 closely follows the "pore network" obtained from following the geodesic network linking the critical points described in Sect. 2. The interface position associated with constricted regions in saddle-points acts as a capillary barrier for the liquid-gas interface.

The invasion percolation model can be tested on a broader scale. Figure 5a, c, e shows the experimental invading patterns obtained at different times with Fig. 3 set up, and using the artificial micro-machined rough surface described in Sect. 4. Fig. 5b, d, f shows the numerical prediction using percolation threshold (10) and the algorithm detailed in Sect. 4.1. The comparison between the Fig. 5a, c, e and b, d, f shows a good agreement between the experimental observation and the numerical computations. There are nevertheless differences, that can, for example, be observed between Fig. 5e and f. Such difference is nevertheless self-consistent with the previously mentioned mismatch between the experimental aperture field and the numerical model. Let us first comment about the impact of the in-plane curvature expression 

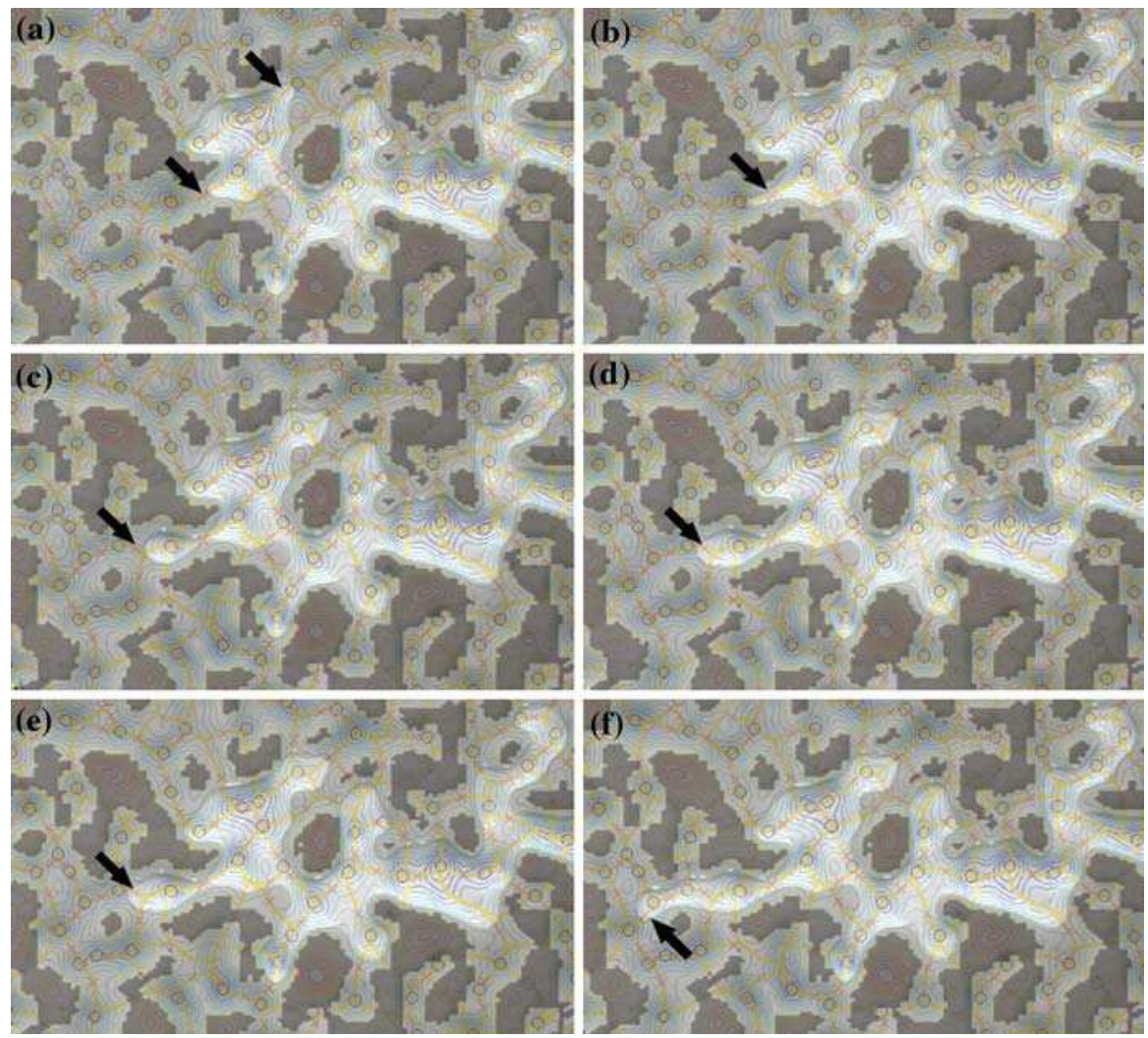

Fig. 4 Each figure displays the superposition of three objects. The first one is the aperture level set computed from randomly generated short-correlated surfaces from which one can distinguish the aperture maxima (with circles) and saddle-points (with crosses). Dark regions are contact regions between the upper and the lower surface. The second object (in yellow) is the geodesic network linking maxima through their associated saddle-point. The third object is the experimental liquid-air interface, visualized at different times for which clear regions are the gas phase. As time is increased the air is invading the pore space through "largest" pores. The two arrows displayed in (a) show the saddle-points from which invasion occurs in (b). Same conventions from (c) to (d), and from (e) to (f) where the last arrow indicates the next saddle to be invaded

obtained in (10) as compare to the simple model (1). To this end, simulations using an invasion criterion that only takes into account the local aperture of saddle-point $\Delta P_{t h}=2 \gamma / h_{0}$ have been compared to simulations using (10) for several numerical random realizations, using the same contact surfaces equal to approximately 0.3 No significant difference was observed. Then we explored numerically systems closer to the percolation threshold considering again several realizations. The geometrical percolation threshold for the system under study has been studied by Mourzenko et al. (1996) and is close to 0.5 for large systems. An interesting observation came out from these simulations. Occasionally, i.e., for some but not all realizations, the invasion path was different when the in-plane curvature was neglected. This can be understood as follows. Far from the percolation threshold, numerous saddle-points are available for the invasion and the hierarchy of available saddle-points at the liquid/gas interface is unambiguously given by the first-order capillary pressure threshold approximation. Closer to the percolation threshold, the list of open saddle-points on the percolation cluster skeleton is far more limited. It becomes then possible that the invading fluid has to choose only between 
two saddle-points to continue the invasion. When it happens that these two saddle-points have practically the same aperture, the in-plane curvature correction contribution has a significant impact on the choice of the saddle-point to be invaded, resulting in a significantly different invasion path depending on the consideration or not of the in-plane curvature correction. Thus, it can be concluded that, for certain realizations, the in-plane curvature at the saddle-point can have a significant impact on the invasion criteria, when the saddle-point constrictions are tight, so that the contact area is close to 0.5 . Another interesting point concerns the perfectly wetting conditions that have been assumed all along this paper. The analysis presented here is easy to adapt to more general wettability conditions. Suppose the contact angles are $\theta$ on top surface and $\theta^{\prime}$ on bottom surface. Then as $\nabla h=0$ at the saddle-point, i.e., the surfaces are locally parallel, a simple calculation shows that the in-depth curvature radius at the saddle-point can be found equal to $h /\left(\cos \theta+\cos \theta^{\prime}\right)$. Thus the invasion criteria (10) can be extended to the leading order to heterogeneous wetting conditions from simply replacing the constant $\cos \theta$ on the right hand side by $\left(\cos \theta+\cos \theta^{\prime}\right) / 2$. The appendix analyzes more deeply the equivalence of this criteria for describing drainage between two rough surfaces, between one rough surface and a flat plane.

\section{Conclusion}

In this paper, we studied quasi-static drainage in a system formed by two short-correlated rough surfaces. We showed that this displacement process in Gaussian fracture can be modeled using a standard IP algorithm on a random network. The random network is constructed from the identification of the fracture aperture field critical points (maxima and saddlepoints). In particular, the network has a variable coordination number and can be viewed as the topological skeleton of the aperture field. This network is designed for systems with significant contact areas so as to have well defined constrictions (at the saddle-points) between valleys. As presented in details in (Plouraboué et al. 2006) and (Flukiger et al. 2006), this bond network is also very well adapted to the determination of the fracture permeability and conductivity. Thus it can be surmised that this network representation will be useful for modeling other transport processes between short correlated rough surfaces.

Acknowledgements The research presented in this paper has been supported by GDR 2345 "Etanchéité statique par joints métalliques en conditions extrêmes" and encouraged by CNES, Snecma-Moteur, EDF and CNRS. We thank G.Dhoye, F. Esteban and S.Cazin for technical supports.

\section{Appendix}

In this appendix we analyze the static shape of a interface pined between two rough surfaces in two dimensions having heterogeneous contact angles. From this static shape we deduce the radius of curvature of the interface as a function of the local slopes and contact angles.

We denote $\beta, \beta^{\prime}$ the upper and lower angle associated with the surface slopes $\tan \beta=d Z_{2} / d x$ and $\tan \beta^{\prime}=-d Z_{1} / d x . \theta, \theta^{\prime}$ are the respective contact angles on upper and lower surface as depicted on Fig. 6 .

Let us now deduce the radius of curvature. We first establish different intermediate equalities.

From Fig. 7a, on can see that $\widehat{B D C}=2 \gamma^{\prime}$, where $B$ and $C$ are the contact line points of the interface on the upper and lower surface, and $D$ is the center of the radius of curvature. 

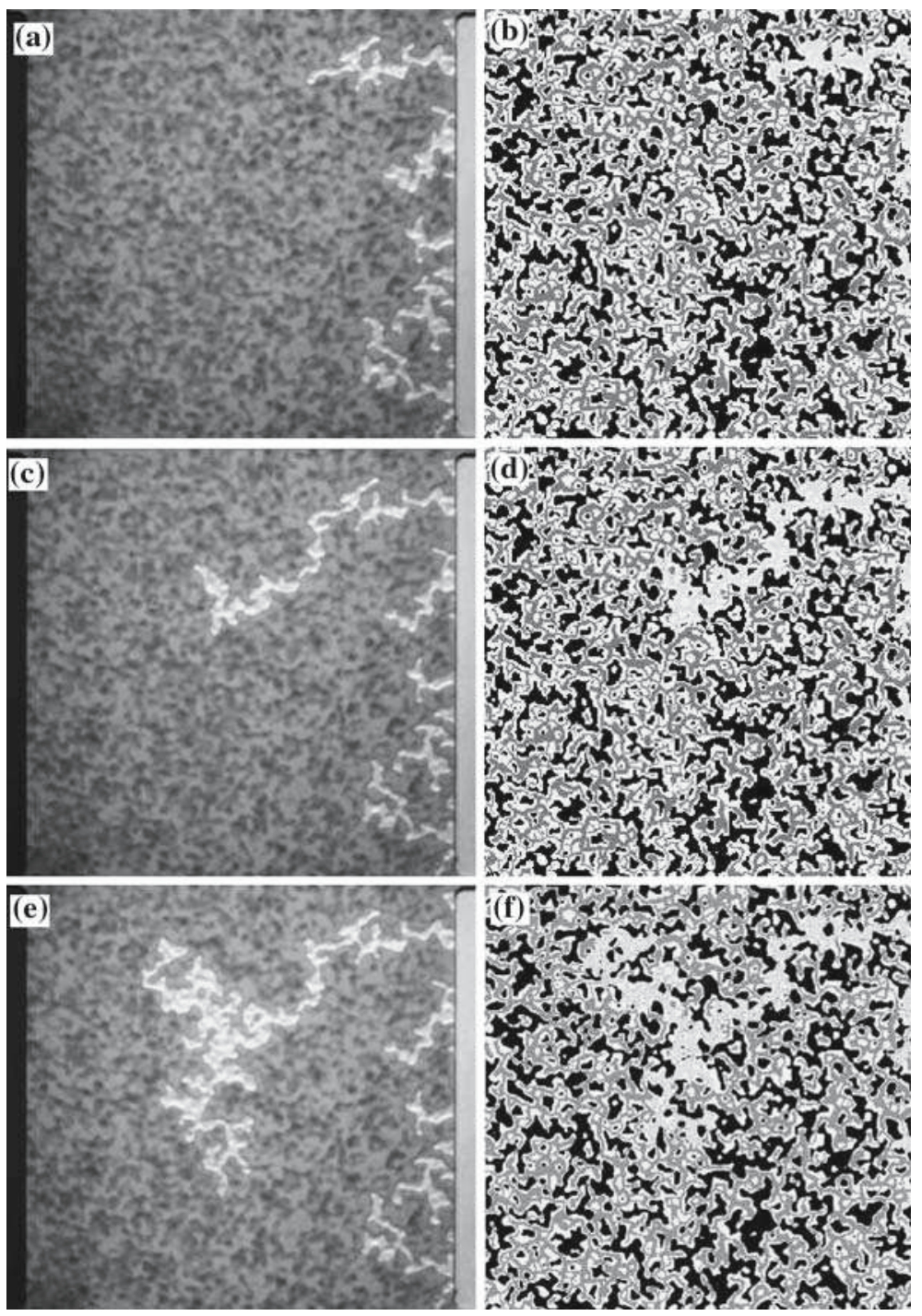

Fig. 5 (a, c, e) Displacement patterns observed during the slow drainage experiment within artificial fracture. Air (white) displaces wetting oil (gray). (b, d, f) Simulated displacement patterns using the IP model on the aperture saddle-point network

From Fig. 7a, one can also deduce a simple equality between all the angles:

$$
2 \gamma^{\prime}+\theta+\theta^{\prime}+\beta+\beta^{\prime}=\pi
$$

since furthermore, $\sin \gamma^{\prime}=B C / 2 R$, then

$$
\sin \left(\frac{\theta+\theta^{\prime}+\beta+\beta^{\prime}}{2}\right)=\frac{B C}{2 R}
$$


Fig. 6 Sketch of a pinned interface between two rough surfaces having local splopes $\beta, \beta^{\prime}$ and contact angles $\theta, \theta^{\prime}$

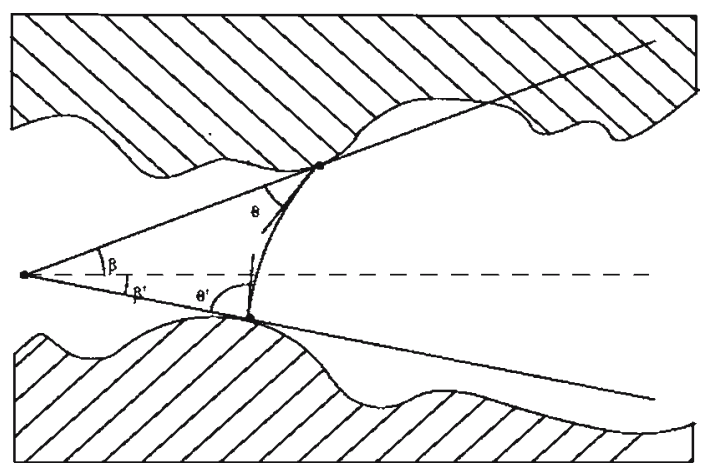

(a)

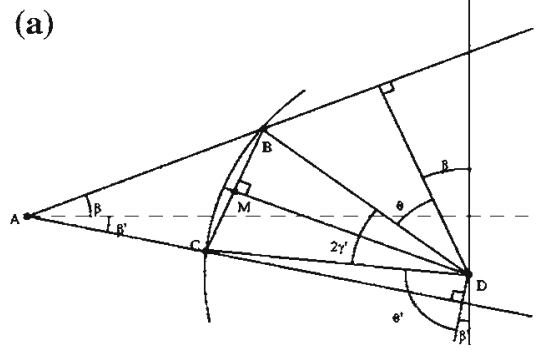

(c)

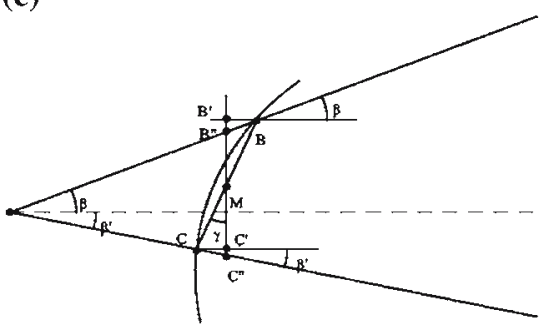

(b)

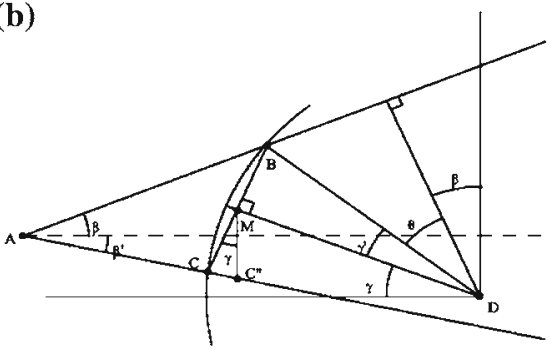

(d)

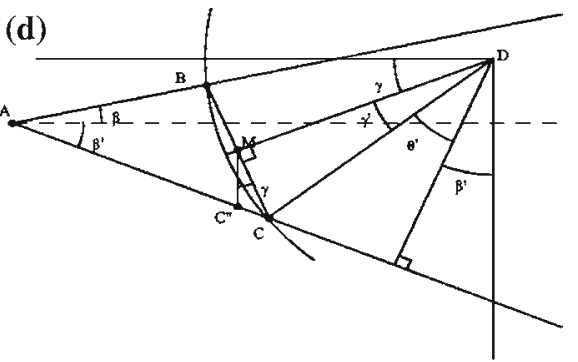

Fig. 7 Different mathematical cases considered in the appendix associated with the physical situation depicted in Fig. 6

The aperture between the two rough surface is given by $B^{\prime \prime} C^{\prime \prime}$ (cf. Fig. 7c) that we will denote $h=B^{\prime \prime} C^{\prime \prime}$ in the following. To compute this local aperture $h$, let us first consider the angle $\gamma=\widehat{C M C^{\prime \prime}}$ (clockwise oriented) of the $B C$ cord with vertical direction (cf. Fig. 7b). Two cases need then to be considered:

i. $\gamma>0$,

ii. $\gamma<0$,

In the first case (i), Fig. 7b has to be considered, for which the following identity applies :

$$
\gamma+\gamma^{\prime}+\theta+\beta=\pi / 2
$$

so that with (11), this leads to:

$$
\gamma=\frac{1}{2}\left(\beta^{\prime}-\beta+\theta^{\prime}-\theta\right)
$$


Then, using notation of Fig. 7b,

$$
\cos \gamma=\frac{B^{\prime} C^{\prime}}{B C}=\frac{h+B^{\prime} B^{\prime \prime}-C^{\prime} C^{\prime \prime}}{B C}
$$

But realizing that:

$$
\left.\begin{array}{rl}
\widehat{C^{\prime \prime} C C^{\prime}} & =\beta^{\prime} \Rightarrow \tan \beta^{\prime}=\frac{C^{\prime} C^{\prime \prime}}{C C^{\prime}} \\
\widehat{C M C^{\prime}} & =\gamma \Rightarrow \sin \gamma=\frac{C^{\prime} C}{B C / 2}
\end{array}\right\} C^{\prime} C^{\prime \prime}=\tan \beta^{\prime} \sin \gamma \frac{B C}{2}
$$

equivalently for $B^{\prime} B^{\prime \prime}$ Eqs. 15 and 16 lead to

$$
B C=\frac{h}{\cos \gamma+\left(-\tan \beta+\tan \beta^{\prime}\right) \sin \gamma}
$$

In the second case (ii) Fig. $7 \mathrm{~d}$ should be considered so that one can realize that, then

$$
-\gamma+\gamma^{\prime}+\theta^{\prime}+\beta^{\prime}=\pi / 2
$$

so that from (11), this leads to:

$$
\gamma=\frac{1}{2}\left(\beta^{\prime}-\beta+\theta^{\prime}-\theta\right)
$$

Following the same steps, one realizes that in this case

$$
\cos \gamma=\frac{B^{\prime} C^{\prime}}{B C}=\frac{h-B^{\prime} B^{\prime \prime}+C^{\prime} C^{\prime \prime}}{B C}
$$

as well as,

$$
\left.\begin{array}{l}
\widehat{C^{\prime \prime} C C^{\prime}}=\beta^{\prime} \Rightarrow \tan \beta^{\prime}=\frac{C^{\prime} C^{\prime \prime}}{C C^{\prime}} \\
\widehat{C M C^{\prime}}=\gamma \Rightarrow-\sin \gamma=\frac{C^{\prime} C}{B C / 2}
\end{array}\right\} C^{\prime} C^{\prime \prime}=-\tan \beta^{\prime} \sin \gamma \frac{B C}{2}
$$

Equations 20 and 21 lead to

$$
B C=\frac{h}{\cos \gamma+\left(-\tan \beta+\tan \beta^{\prime}\right) \sin \gamma}
$$

Hence, in both cases (i) and (ii) one has:

$$
\gamma=\frac{1}{2}\left(\beta^{\prime}-\beta+\theta^{\prime}-\theta\right)
$$

and,

$$
B C=\frac{h}{\cos \gamma+\left(-\tan \beta+\tan \beta^{\prime}\right) \sin \gamma}
$$

Using (12), (24), and (23) then one gets finally the expression for the local radius of curvature $R$ of the interface:

$$
R=\frac{h}{2} \frac{1}{\cos \frac{\beta+\beta^{\prime}+\theta+\theta^{\prime}}{2}\left[\cos \frac{\beta^{\prime}-\beta+\theta^{\prime}-\theta}{2}+\left(-\tan \beta+\tan \beta^{\prime}\right) \sin \frac{\beta^{\prime}-\beta+\theta^{\prime}-\theta}{2}\right]}
$$

It is interesting to look at the approximation of this radius of curvature as a function of the local slopes $\beta$ and $\beta^{\prime}$ when they are both small, as usually considered in the context of the lubrication approximation. In this case one finds that:

$$
R=h\left(\frac{1}{\cos \theta+\cos \theta^{\prime}}-\frac{\left(\beta \sin \theta^{\prime}+\beta^{\prime} \sin \theta\right)}{\left(\cos \theta+\cos \theta^{\prime}\right)^{2}}\right)+O\left(\beta^{2}, \beta^{\prime 2}\right)
$$


This last result shows that, to the leading order, the radius of curvature only depends on the contact angles $\theta$ and $\theta^{\prime}$. The first small slope correction displays a linear dependence with the surface slopes $\beta$ and $\beta^{\prime}$. In the case of symmetrical wettability, one can see that this correction involves the sum of the angles. Hence, if one consider the equivalent situation of a single rough surface having local slope $\beta+\beta^{\prime}$ resulting from taking the height difference between $Z_{2}$ and $Z_{1}$ surfaces and a flat surface, the radius of curvature will be exactly identical to the small slope approximation found in (26). Hence in both situation the invasion capillary pressure is the same, and thus the drainage problem is equivalent. In the case of heterogeneous wettability, one can still find a equivalence between the rough/flat case with the rough/rough situation from properly weighting the surface height $Z_{2}$ and $Z_{1}$ with wetting angles $\sin \theta^{\prime}$ and $\sin \theta$. Moreover, since the invasion criteria is written on critical points for which the local slope is zero, in this case $\beta+\beta^{\prime}=0$ and the invasion criteria only depends on the first term of expression (26) from neglecting $O\left(\beta^{2}, \beta^{2}\right)$ terms self-consistently with lubrication approximation.

\section{References}

Adler, P.M., Thovert, J.F.: Fractures and fracture networks. Kluwer Academic Publishers, Amsterdam (1999) Amundsen, H., Wagner, G., Oxaal, U., Meakin, P., Feder, J., Jossang, T.: Slow two-phase flow in artificial fractures: experiments and simulations. Wat. Res. Res. 35, 2619-2626 (1999)

Amyot, O., Plouraboué, F.: Capillary pinching in a pinched micro-channel. Phys. Fluids 19, 033101 (2007)

Berkowitz, B.: Characterizing flow and transport in fractured geological media : a review. Adv. Wat. Res. 25, 861-884 (2002)

Blunt, M.J.: Flow in porous media: pore networks models and multiphase flows. Curr. Opin. Colloid Interface Sci. 6, 187-207 (2001)

Blunt, M.J., Jackson, M.D., Piri, M., Valvatne, P.H.: Detailed physics, predictive capabilities and macroscopic consequences for pore-network models of multiphase flow. Adv. Wat. Res. 25, 1069-1089 (2002)

Bories, S., Prat, M.: Isothermal Nucleation and Bubble Growth in Porous Media at Low SupersaturationsTransport Phenomena in Porous Media, pp. 276-315. D.B. Ingham et K. Lambert Ed., UK (2002)

Brown, S.R., Scholz, C.H.: Broad bandwidth study of the topography of natural rock surfaces. J. Geoph. Res. 90, 12-575-12-582 (1985)

Bryant, S.L., Mellor, D.W., Cade, C.A.: Physically representative network models of transport in porous media.. AIChE J. 39(3), 387-396 (1993)

Chrysikopoulos, C.V., Abdel-Salam, A.: Modeling colloid transport and deposition in saturated fractures. Colloids Surfaces A: Physicochem. Eng. Aspects 121, 189-202 (1997)

Chrysikopoulos, C.V., James, S.C.: Transport of neutrally buoyant and dense variably sized colloids in a two-dimensional fracture with anisotropic aperture. Trans. Porous Med. 51, 191-210 (2003)

Evans, D.D., Nicholson, T.J., Rasmussen, T.E.C.: Flow and Transport Through Unsaturated Fractured Rock. AGU Geophysical Monograph Series, vol 42, New York (2001)

Faybishenko, B., Witherspoon, P.A., Benson, S.M.: Dynamics of Fluid I Fractured Rock. AGU Geophysical Monograph Series, vol 122, New York (2000)

Flukiger, F., Plouraboué, F., Prat, M.: Non-universal conductivity exponents in continuum percolating Gaussian fracture. in revision for Phys. Rev. E (2006)

Fourar, M., Bories, S., Lenormand, R., Persoff, R.F.: Two-phase flow in smooth and rough fractures: measurement and correlation by porous media and pipe-flow models. Wat. Res. Res. 29(11), 3699-3708 (1993)

De Gennes, P.G.: Theory of slow biphasic flow in porous media. Phys. Chem. Hydrol. 4, 175-185 (1983)

Geoffroy, S., Prat, M.: On the leak through a spiral-groove metallic static ring gasket. ASME J. Fluids Eng. 126(1), 48-54 (2004)

Geoffroy, S., Plouraboué, F., Prat, M., Amyot, O.: Quasi-static liquid-air drainage in narrow channels with variations in the gap. J. Colloids Interface Sci. 294, 165-175 (2006)

Glass, R.J., Nicholl, M.J., Yarrigton, L.: A modified invasion percolation model for low-capillary number immiscible displacements in horizontal rough-walled fractures: influence of local in-plane curvature. Wat. Res. Res. 34(12), 3215-3234 (1998)

Glass, R.J., Rajaram, H., Nicholl, M.J., Detwilder, R.L.: The interaction of two fluid phases in fractured media. Curr. Opin. Colloid Interface Sci. 6, 223-235 (2001) 
Lenormand, R., Touboul, E., Zarcone, C.: Numerical models and experiments on immiscible displacements in porous media. J. Fluid Mech. 189, 165-187 (1988)

Letalleur, N., Plouraboué, F., Prat, M.: Average flow model of rough surface lubrication: flow factors for sinusoidal surfaces. ASME J. Tribol. 124, 539 (2002)

Loggia, D., Gouze, P., Greswell, R., Parker, D.J.: Investigation of the geometrical dispersion regime in a single fracture using positron emission projection imaging journal transport in porous media. Trans. Por. Med. 55(1), 1-20 (1995)

Makse, H.A., Havlin, S., Schwartz, M., Stanley, H.E.: Method for generating long-range correlation for large systems. Phys. Rev. E 53(5), 5445 (1996)

Mendoza, C.A., Sudicki, E.A.: Hierarchical scaling of constitutive relationships controlling multi-phase flow in fractured geologic media, in reservoir chracterization. In: 3rd International Technical Conference: Papers, Pennwell, Tulsa, Okla., B.Linville (1991)

Mourzenko, V.V., Thovert, J.F., Adler, P.M.: Geometry of simulated fractures. Phys. Rev. E 53(6), 56065626 (1996)

National Research council: Rock Fractures and Fluid Flow: Contemporary Understanding and Applications. Technical Report, Washington D.C.: National Academic Press., Avril (1996)

Pereira, G.G., Pinczewski, W.V., Chan, D.Y.C., Paterson, L., Oren, P.E.: Pore-scale network model for drainage-dominated three-phase flow in porous media. Trans. Por. Med. 24(2), 167-201 (1996)

Plouraboué, F., Flukiger, F., Prat, M., Crispel, P.: Geodesic network method for flows between two rough surfaces in contact. Phys. Rev. E 73, 036305 (2006)

Plouraboué, F., Geoffroy, S., Prat, M.: Conductances between confied rough walls. Phys. Fluids 16(3), 615624 (2004)

Plouraboué, F., Kurowski, P., Hulin, J.P., Roux, S., Schmittbuhl, J.: Aperture of rough cracks. Phys. Rev. E 51(3), 1675-1685 (1995)

Plouraboué, F., Prat, M., Letalleur, N.: Sliding lubricated anisotropic rough surfaces. Phys. Rev. E 64(1), 011202 (2001)

Polycarpou, A., Etsion, I.: A Model for satic sealing performance of compliant metallic gas seals including surface roughness and rarefaction effects. Tribol. Transac. 43(2), 237-244 (2000)

Prat, M.: Recent advances in pore-scale models for drying of porous media. Chem. Eng. J. 86(1-2), 153164 (2002)

Prat, M., Letalleur, N., Plouraboué, F.: Averaged Reynolds equation for flow between rough surfaces in sliding motion. Trans. Por. Med. 48, 291-313 (2002)

Sahimi, M.: Flow and Transport in Porous Media and Fractured Rock. VCH Wienheim, New York (1995)

Satik, C., Yortsos, Y.C.: A pore network study of bubble growth in porous media driven by heat transfer. J. Heat Trans. T. ASME 118, 455-462 (1996)

Satik, C., Li, X., Yortsos, Y.C.: Scaling of single bubble growth in a porous medium. Phys. Rev. E 51, 3286 (1995)

Sok, R.M., Knackstedt, M.A., Sheppard, A.P., Pinczewski, W.V., Lindquist, W.B., Venkatarangan, A., Paterson, L. : Direct and stochastic generation of network models from tomographic images; effect of topology on residual saturations. Trans. Por. Med. 46, 2-3 345-371 (2002)

Tarjan, R.: Data Structures and Network Algorithms. Society for Industrial and Applied mathematics, NewYork, USA (1983)

Vandersteen, K., Carmeliet, J., Feyen, J.: A network modeling approach to derive unsaturated hydraulic properties of a rough -walled fracture. Trans. Por. Med. 50, 197-221 (2003)

Wagner, G., Meakin, P., Feder, J., Jossang, T.: Invasion percolation on self-affine topographies. Phys. Rev. E 55(2), 1698-1703 (1997)

Weinrib, A.: Percolation threshold of two-dimensional continuum system. Phys. Rev. B 26(3), 13521361 (1982)

Wilkinson, D.: Percolation model of immiscible displacement in the presence of buoyancy forces. Phys. Rev. A 30, 520-531 (1984)

Wilkinson, D., Willemsen, J.F.: Invasion percolation: a new form of percolation theory. J. Phys. A-Math. Gen. 16, 3365-3376 (1983) 